\title{
Gaps between antibiotic prescribing practices and international guidelines for women undergoing caesarean deliveries in rural Rwanda: A retrospective cohort study
}

\author{
Fredrick Kateera ${ }^{1}$, Kara Faktor ${ }^{2}$, Theoneste Nkurunziza ${ }^{3}$, Teena Cherian ${ }^{2}$, Bethany \\ Hedt-Gauthier $^{2}$, Laban Bikorimana ${ }^{3}$, Jonathan Nkurunziza ${ }^{3}$, Rachel Koch ${ }^{2}$, Patient \\ Ngamije $^{4}$, and Robert Riviello ${ }^{2}$ \\ ${ }^{1}$ Partners In Health / Inshuti Mu Buzima \\ ${ }^{2}$ Harvard Medical School \\ ${ }^{3}$ Partners in Health / Inshuti Mu Buzima \\ ${ }^{4}$ Republic of Rwanda Ministry of Health
}

May 6, 2020

\begin{abstract}
Objective: Antibiotic stewardship remains a significant challenge in sub-Saharan Africa, with paucity of systematic data on antimicrobial use in surgical care. We characterise antibiotic prescribing practices for women who delivered via caesarean section (c-section) in rural Rwanda. Design: Nested retrospective cohort study. Setting: Kirehe District, rural Eastern Rwanda. Population: All women aged > 18 years who delivered by c-section between November 2017 and February 2018. Methods: Data on antibiotic use, including name, indication, dose/frequency/route, time, and duration of administration were extracted from patient charts. Prescribing practices statistics were summarised by the timing and type. Main Outcome Measures: Preoperative and post-operative antibiotic prescriptions, by time, name and dose. Results: A total of 506 patients were enrolled. $80.8 \%$ received a pre-operative antibiotic, with $67.4 \%$ receiving an antibiotic within one hour of incision. The most prevalent pre-operative antibiotics prescribed were Ceftriaxone (83.3\%) and Ampicillin (15.0\%). Post-operatively during hospitalisation, 95.7\% of patients received two antibiotics, most commonly Ampicillin (99.2\%) in combination with Gentamicin (98.5\%). For all but one, antibiotics were prescribed without indication of infection. At discharge, while $27.8 \%$ of patients were not prescribed any additional antibiotics, $72.2 \%$ were prescribed at least one antibiotic. Conclusions: Large deviations in antibiotic prescribing practices as compared to international prophylaxis guidelines were observed. Further studies to understand the rationale for these deviations and the effects of over-prescription on infection prevention and antibiotic resistance are needed to support context-appropriate guidelines in Rwanda and in comparable settings. Funding: NIH R21EB022369 Keywords: Antimicrobial use, sub-Saharan Africa, global surgery
\end{abstract}

\section{Introduction}

Globally, approximately 5 million cases of pregnancy-related infection occur annually, of which 75,000 result in death. ${ }^{1}$ Risk of developing a post-partum infection is much higher in low- and middle-income countries (LMICs) due to poor hygiene conditions coupled with limited resources. ${ }^{1,2}$ While access to caesarean sections (c-sections) in sub-Saharan Africa (SSA) has improved, associated surgical site infections (SSIs) present a new challenge. SSIs are one of the most common healthcare-associated infections worldwide. ${ }^{3}$ Given that c-sections comprise a large portion of surgical care in SSA, they account for a large portion of SSIs in this setting. ${ }^{4-6}$

Pre-operative antibiotic prophylaxis reduces post-caesarean infection rates, ${ }^{7}$ with one systematic review 
reporting a $39 \%$ decrease in SSI incidence among those receiving antibiotic prophylaxis. ${ }^{8}$ Several studies, including randomized control trials (RCTs), conducted in resource-limited settings suggest non-inferiority of single dose pre-surgical prophylaxis compared to prolonged antibiotic administration. ${ }^{9-11}$ Yet, rational antibiotic use remains a significant challenge in SSA where there are limited antimicrobial stewardship-related trainings, compliance processes, and on-site access to data-informed prescribing protocols that collectively support effective, context-specific antibiotic use practices. ${ }^{12}$

According to the World Health Organization (WHO) global guidelines for the prevention of SSIs, antibiotic prophylaxis should be administered within 120 minutes before the first incision without prolongation after completion of the operation. ${ }^{13}$ Locally, the Rwandan Ministry of Health (RMOH) protocol published in 2012 recommends use of Ampicillin for c-section pre-operative prophylaxis, but doesn't specify timing. ${ }^{14}$ At the hospital where the study was conducted, anecdotal reports indicate general practitioners (GPs) follow Society for Healthcare Epidemiology of America (SHEA)/Infectious Disease Society of America (IDSA) guidelines, which recommend administration of intravenous antibiotics within 1 hour of incision to maximize tissue concentration. ${ }^{15}$ Inconsistences in guidance may partly account for variation in antibiotic prescribing.

In SSA, SSI incidence among women undergoing a c-section ranges from 7.1-12\%. ${ }^{7}, 16$ Our group recently reported a post-caesarean SSI rate of $10.9 \%$ at the district hospital involved in this study. ${ }^{17}$ While there is limited information on pathogens and antibiotic sensitivity within SSA, ${ }^{18-22}$ especially with respect to post-caesarean wound infections, ${ }^{23-26}$ existing data suggest emerging antimicrobial resistance. However, to our knowledge, no study has focused on rural district hospitals. Moreover, there is a paucity of systematic data on antibiotic prescribing practices aimed at SSI prevention. ${ }^{22,27,28}$ Here, we characterize perioperative antibiotic prescriptions for women who underwent a c-section at a rural Rwandan district hospital to better understand prescribing practices as they relate to international protocols.

\section{Methods}

Study data were collected as part of an ongoing prospective study to evaluate the impact of a mobile health technology (mHealth)-community health worker (CHW) intervention on detection of women with suspected post-caesarean SSIs and their subsequent return to a health centre or hospital for management. ${ }^{29}$ The prospective mHealth-CHW study was funded by the National Institute of Health (NIH R21EB022369) and included external peer review for scientific quality. Patients were not involved in study design.

\section{Study Site}

The study was conducted in rural Eastern Rwanda at Kirehe District Hospital (KDH), which provides maternal and child health services to a catchment area of approximately 370,000 people. In 2017, 1,465 women delivered by c-section at KDH. The vast majority of c-sections are performed by the $14 \mathrm{GPs}$, with a few performed by a resident obstetrician at $\mathrm{KDH}$. In the absence of complications, a mother is routinely discharged on post-operative day three and instructed to attend a follow-up visit at her local health centre for wound care. We analysed use of antibiotics pre-operatively relative to the hospital leadership-reported protocol of administering antibiotic prophylaxis within 1-hour prior to incision, similar to the United States SHEA/IDSA recommendations. ${ }^{15}$

\section{Data Collection and Analysis}

All women aged [?]18 years, who are residents in Kirehe district and underwent a c-section at KDH between November 2017 and February 2018 were eligible for inclusion. In the parent study, women living in the Mahama refugee camp who delivered by c-section at KDH were excluded from study enrolment because camp care protocols would not allow the women to comply with our study protocols. For all eligible patients, a signed written informed consent was obtained before patient enrolment. At enrolment, study-trained data collectors administered a sociodemographic survey and subsequently, at discharge, performed clinical chart review where data on antibiotic(s) administered, indication, dose/frequency/route, timing and duration of administration were extracted. Descriptive statistics are used to summarize socio-demographic and clinical data. Data on prescribing practices are presented as frequencies and percentages, and stratified by time of 
antibiotic prescription (pre-operative, post-operative during admission, and at discharge).

\section{Ethics}

All eligible women were read and provided with a copy of a written informed consent document in the local language (Kinyarwanda), and invited to provide a signed written consent prior to study enrolment. Data were collected using encrypted study tablets and entered directly into Research Electronic Data Capture (REDCap), a secure password-protected database. ${ }^{30}$

\section{Results}

Of the 506 women enrolled, $357(70.6 \%)$ were aged between 18 and 30 years (Table 1$)$. Most were either married $(\mathrm{n}=228,45.1 \%)$ or living with a partner $(\mathrm{n}=228,45.1 \%)$, had at least a primary education $(\mathrm{n}=443$, $87.5 \%)$ and reported farming $(\mathrm{n}=356,70.4 \%)$ as their principle occupation. For $453(89.5 \%)$ women, the reported monthly household income was less than 30,000 RWF (approximately 33.30 USD).

Nearly all women underwent a c-section for either emergent indications $(\mathrm{n}=140,27.7 \%)$ - requiring an immediate intervention - or urgent indications $(\mathrm{n}=355,70.2 \%)$, for which the patient could wait for intervention until she was medically stable. The indications for a c-section included foetal distress $(\mathrm{n}=174,34.4 \%)$ and a previous c-section scar $(\mathrm{n}=160,31.6 \%)$ as most prevalent, followed by cephalopelvic disproportion $(\mathrm{n}=82$, $16.2 \%)$, prolonged or obstructed labour $(\mathrm{n}=60,11.9 \%)$ and malpresentation $(\mathrm{n}=54,10.7 \%)$.

A majority $(\mathrm{n}=409,80.8 \%)$ of the 506 enrolled patients received a pre-operative antibiotic (Table 2). Of these, $239(58.4 \%)$ had data on timing of the pre-operative dose, with $161(67.4 \%)$ receiving the antibiotic within an hour prior to incision. The median time between antibiotic prophylaxis and the surgical incision was 40 minutes (interquartile range (IQR): 25, 74 minutes). For the 409 women who received pre-operative prophylaxis, the antibiotics most frequently prescribed were Ceftriaxone ( $\mathrm{n}=343,83.9 \%)$ and Ampicillin $(\mathrm{n}=62,15.2 \%)$-almost exclusively as a single dose $(\mathrm{n}=405,99.3 \%)$.

After surgery and before hospital discharge, 485 (98.6\%) of the 492 patients with post-operative prescription data, were given at least one antibiotic (Figure 1). Apart from one patient who received therapeutic antibiotics for a surgical site infection, all antibiotics $(n=484,99.8 \%)$ were prescribed for prophylaxis only . The median time between surgery completion and the first post-operative dose was 15.6 hours (IQR: 10.9, 21.7 hours). Of the 485 patients who received post-operative antibiotics, most were prescribed two antibiotics $(\mathrm{n}=464,95.7 \%)$. Ampicillin was the most commonly prescribed $(\mathrm{n}=481,99.2 \%)$, and almost always in combination with Gentamicin $(\mathrm{n}=468,98.5 \%)$. Ten patients $(2.1 \%)$ received only one antibiotic and another ten $(2.1 \%)$ patients received three antibiotics. Among those who received a third antibiotic, four received Metronidazole, five received Gentamicin and one received Cloxacillin.

At discharge, 134 (27.8\%) of the 482 patients with discharge antibiotic prescription data were not prescribed any antibiotics (Figure 2). Among patients who received antibiotics at discharge, 332 (96.2\%) patients were prescribed only one antibiotic, while $13(3.8 \%)$ patients were prescribed two antibiotics. The most prevalent monotherapies prescribed upon discharge were Amoxicillin $(\mathrm{n}=265,76.1 \%)$ and Cloxacillin $(\mathrm{n}=74$, $21.3 \%$ ). Among the 13 patients who were prescribed two antibiotics at discharge, 11 (84.6\%) were prescribed Metronidazole as one of the two antibiotics.

Discussion

\section{Main Findings}

In our study, antibiotic prescribing at KDH showed noteworthy divergence from the SHEA/IDSA recommendations for the prevention of SSIs in acute care hospitals. ${ }^{15}$ This divergence is consistent with the 29-country WHO Multicountry Survey on Maternal and Newborn Health which found that $34.3 \%$ of included facilities in Africa, Asia, Latin America and the Middle East reported less than $90 \%$ pre-surgical prophylaxis coverage. ${ }^{31}$ A recent systematic review on c-sections in SSA, which summarized 17 studies that reported on antibiotic prescribing practices, similarly noted divergence from the WHO's SSI prevention guidelines. ${ }^{32}$ Deviations noted in our study included under-use of pre-operative antibiotics, with only $67.4 \%$ of our study participants 
having a pre-operative antibiotic prescribed within an hour prior to the first incision. Of concern, nearly all patients received two antibiotics after surgery and before discharge, while the WHO guidelines indicate none should be prescribed in the absence of indications of infection.

One challenge for pre-operative antibiotics is that neither KDH nor RMOH obstetrics and gynaecology guidelines specify the timing of these antibiotics during c-section care. ${ }^{14}$ While this study did not explore reasons for failure to administer pre-operative antibiotics, or for the timing deviations, lack of local guidelines and inconsistencies in guidelines released by entities like the WHO, CDC and SHEA/IDSA may contribute to the observed variations. In fact, in the multicountry survey conducted by the WHO, coverage of pre-surgical prophylaxis was greater in facilities using local and WHO guidelines, as well those reporting clinical audits of antibiotic prescribing practices. ${ }^{31}$

Our observation of over-prescription of antibiotics post-operatively has been noted elsewhere in SSA, ${ }^{33,34}$ and internationally, ${ }^{35}$ showing high rates of poorly-timed pre-surgical antibiotic doses with prophylaxis often extending beyond the operative period. In a study at a Sudanese hospital where an overall SSI rate of $7.8 \%$ was reported, a lack of evidence-based guidelines and fear of the patient developing SSI complications were suggested as possible contributors to extended post-operative prescribing by healthcare providers. ${ }^{33} \mathrm{~A}$ followup study in this setting is currently underway.

Beyond the timing and duration of the antibiotics prescribed, selection of an antibiotic with appropriate coverage and half-life is also important in achieving adequate pre-operative prophylaxis. At $\mathrm{KDH}$, Ceftriaxone (83.9\%) was overwhelmingly the most frequently prescribed pre-operative antibiotic, followed by Ampicillin $(15.2 \%)$, the antibiotic recommended in Rwanda's national obstetrics and gynaecology guidelines. ${ }^{14}$ Reasons for the predominate use of Ceftriaxone were not elucidated in this study. Such discrepancies may partly be explained by a GP's training-based prescribing habits, a consequence of antibiotic availability, a preference for oral rather than invasively provided medications or differential pharma-driven marketing influences. A further exploration of influencers of antibiotic prescribing habits is needed.

When compared to international guidelines, antibiotics were over-used in this study with respect to prolonged duration, too broad a spectrum of antimicrobial coverage or use of multiple antimicrobial agents, as reported elsewhere. ${ }^{33,35}$ What is not clear is whether international guidelines - based predominantly on data from high-income countries - are optimized for settings such as rural district hospitals in SSA. In support of international guidelines, a recent RCT in rural Tanzania demonstrated non-inferiority of single-dose antibiotic prophylaxis when compared to extended antibiotic administration for patients undergoing a c-section. ${ }^{9}$ Furthermore, despite the high prevalence of post-operative prescribing seen in this study, the $10.9 \%$ SSI rate found during the RCT is comparable to rates seen throughout the region, possibly suggesting no benefit to prolonged antibiotic duration for SSI prevention. ${ }^{17}$ However, a recent study at KDH showed that women delivering via c-section who were hospitalized on a day without running water were significantly more likely to develop a SSI (manuscript in draft), suggesting that infection risk, and therefore effective prophylaxis, may need to extend beyond the first incision in this setting.

\section{Strengths and Limitations}

This study is one of few studies characterizing antibiotic use in the context of c-section care in rural Africa. The study team prospectively enrolled patients, worked closely with the hospital teams and collected highquality data. However, while interpreting study findings, a number of limitations should be considered. Col-

lection of antibiotic-related data was by chart review at the time of discharge, rather than direct recording at the time of administration, which may affect accuracy of timing and proper charting of antibiotics prescribed and administered. Since the data collection occurred as part of a larger prospective study, providers may have been somewhat aware that the study team was looking at SSI rates in their patients, potentially causing an artificial increase in antibiotic prescribing in an effort to keep SSI rates low. Lastly, some of the patient exposures, including the peri-operative antibiotics received, may reflect prescribing practices specific to providers at the district hospital, limiting generalizability.

\section{Interpretation}


There are several considerations for interpreting these results. The first is that the providers deviated from international prescribing protocols due to a failure to have and/or understand such protocols. The second is that providers do not believe these protocols take into consideration the potentially high risk of infection and low likelihood of mothers returning for consultation within these contexts. Understanding these contextual factors is key to informing improvements in prescription practices and compliance to protocols.

\section{Conclusion}

Compared to the SHEA/IDSA international guidelines for women who delivered by c-section, our data show large deviations in antibiotic prescribing practices both pre and post-operatively, as well as among different prescribers. Our findings raise questions on whether insisting on a single pre-operative antibiotic dosing regimen, established based on research in developed settings, oversimplifies the challenges of providing optimal care for women undergoing a c-section in this environment. More data is needed to guide development of area-specific guidelines that are both effective and feasible. Furthermore, studies exploring the rationale of antibiotic prescribing decisions, those assessing the effect of over-prescription on infection prevention, and those tracking evolving antibiotic sensitivity are recommended to guide evidence-based antibiotic prescription practices.

\section{Disclosure of interests}

The authors have no relevant interests to disclose.

\section{Contribution to authorship}

FK, BHG, and RR are the three principal investigators for this study, instigated the original idea for the study, developed the funding proposal, applied for funding, and led study design, implementation, data analysis, and the dissemination of results. KF led the implementation of the antimicrobial resistance work specifically. TN, TC, and RK led the implementation of the larger study in which this study is nested. LB, JN, and TN collected data for use in this analysis. PN provided hospital oversight and assisted in study design and data collection. All authors read and commented on manuscript drafts and approved the final version.

\section{Details of ethics approval}

The study protocol was approved by the Partners in Health (PIH)/Inshuti Mu Buzima (IMB) Research Committee and Rwanda National Health Research Committee, with ethics approvals from Rwanda National Ethics Committee in Kigali, Rwanda (1 November 2016; No. 848/RNEC/2016) and Partners Human Research Committee in Boston, USA (9 September 2017; No. 2016P001943/MGH). The study was also approved by the Rwanda Ministry of Health prior to implementation.

\section{Funding}

This study was funded by the National Institutes of Health (NIH R21EB022369) and supported by Partners In Health (PIH) / Inshuti Mu Buzima (IMB).

\section{Acknowledgements}

We are grateful for the support of Kirehe District Hospital leadership and staff during data collection.

\section{References}

1. Miller AE, Morgan C, Vyankandondera J. Causes of puerperal and neonatal sepsis in resource-constrained settings and advocacy for an integrated community-based postnatal approach. Int J Gynecol Obstet [Internet]. 2013;123(1):10-5. Available from: http://dx.doi.org/10.1016/j.ijgo.2013.06.006

2. Ngonzi J, Bebell LM, Fajardo Y, Boatin AA, Siedner MJ, Bassett I V., et al. Incidence of postpartum infection, outcomes and associated risk factors at Mbarara regional referral hospital in Uganda. BMC Pregnancy Childbirth. 2018;18(1):1-11. 
3. Suetens C, Hopkins S, Kolman J, Hogberg LD. Point prevalence survey of healthcare-associated infections and antimicrobial use in European acute care hospitals. 2012.

4. Nejad SB, Allegranzi B, Syed SB, Ellisc B, Pittetd D. Infections liées aux soins de santé en afrique: Une étude systématique. Bull World Health Organ. 2011;89(10):757-65.

5. Harrison MS, Goldenberg RL. Cesarean section in sub-Saharan Africa. Matern Heal Neonatol Perinatol [Internet]. 2016;2(1):6. Available from: http://mhnpjournal.biomedcentral.com/articles/10.1186/s40748-016$0033-\mathrm{x}$

6. Chu K, Maine R, Trelles M. Cesarean section surgical site infections in sub-Saharan Africa: A multi-country study from Medecins sans Frontieres. World J Surg. 2015;39(2):350-5.

7. Amenu D, Belachew T, Araya F. Surgical site infection rate and risk factors among obstetric cases of jimma university specialized hospital, southwest ethiopia. Ethiop J Health Sci [Internet]. 2011;21(2):91-100. Available from: http://www.pubmedcentral.nih.gov/articlerender.fcgi?artid=3275863\&tool=pmcentrez\&rendertype=abstract

8. Smaill FM GR. Antibiotic prophylaxis versus no prophylaxis for preventing infection after cesarean section (Review). Cochrane Libr. 2014;(10).

9. Westen EHMN, Kolk PR, Van Velzen CL, Unkels R, Mmuni NS, Hamisi AD, et al. Single-dose compared with multiple day antibiotic prophylaxis for cesarean section in low-resource settings, a randomized controlled, noninferiority trial. Acta Obstet Gynecol Scand. 2015;94(1):43-9.

10. Lyimo FM, Massinde AN, Kidenya BR, Konje ET, Mshana SE. Single dose of gentamicin in combination with metronidazole versus multiple doses for prevention of postcaesarean infection at Bugando Medical Centre in Mwanza, Tanzania: a randomized, equivalence, controlled trial. BMC Pregnancy Childbirth [Internet]. 2013;13:123. Available from: http://www.ncbi.nlm.nih.gov/pubmed/23721411\%0Ahttp://www.pubmedcentral.nih.gov/articlerender.fcgi?artid=PMC3681

11. Shaheen S, Akhtar S. Comparison of single dose versus multiple doses of antibiotic prophylaxis in elective caesarian section. J Postgrad Med Inst. 2014;28(1):83-6.

12. van Dijck C, Vlieghe E, Cox JA. Antibiotic stewardship interventions in hospitals in low-and middleincome countries: A systematic review. Bull World Health Organ. 2018;96(4):266-80.

13. World Health Organization (WHO). Global guidelines for the prevention of surgical site infection. J Hosp Infect [Internet]. 2017;95(2):135-6. Available from: http://linkinghub.elsevier.com/retrieve/pii/S0195670116305874

14. Rwanda Ministry of Health. Gynecology and Obstetrics, Clinical Protocols \& Treatment Guidelines. 2012.

15. Anderson DJ, Podgorny K, Berríos-Torres SI, Bratzler DW, Dellinger EP, Greene L, et al. Strategies to Prevent Surgical Site Infections in Acute Care Hospitals: 2014 Update. Infect Control Hosp Epidemiol. 2014;35(6):605-27.

16. Wodajo S, Belayneh M, Gebremedhin S. Magnitude and Factors Associated With Post-Cesarean Surgical Site Infection at Hawassa University Teaching and Referral Hospital, Southern Ethiopia: A Cross-sectional Study. J Heal Sci. 2017;27(3):283.

17. Nkurunziza T, Kateera F, Sonderman K, Gruendl M, Nihiwacu E, Ramadhan B, et al. Prevalence and predictors of surgical-site infection after caesarean section at a rural district hospital in Rwanda. Br J Surg. 2019;106(2):e121-8.

18. Dessie W, Mulugeta G, Fentaw S, Mihret A, Hassen M, Abebe E. Pattern of bacterial pathogens and their susceptibility isolated from surgical site infections at selected referral hospitals, Addis Ababa, Ethiopia. Int J Microbiol. 2016; 
19. Mengesha RE, Kasa BGS, Saravanan M, Berhe DF, Wasihun AG. Aerobic bacteria in post surgical wound infections and pattern of their antimicrobial susceptibility in Ayder Teaching and Referral Hospital, Mekelle, Ethiopia. BMC Res Notes. 2014 Aug 27;7(1).

20. Mulu W, Kibru G, Beyene G, Damtie M. Postoperative nosocomial infections and antimicrobial resistance pattern of bacterial isolates among patients admitted at Felege Hiwot Referral Hospital, Bahirdar, Ethiopia. Ethiop J Health Sci. 2012;22(1):7-18.

21. Seni J, Najjuka CF, Kateete DP, Makobore P, Joloba ML, Kajumbula H, et al. Antimicrobial resistance in hospitalized surgical patients: a silently emerging public health concern in Uganda. BMC Res Notes. 2013;6(298).

22. Ampaire L, Muhindo A, Orikiriza P, Mwanga-Amumpaire J, Bebell L, Boum Y. A review of antimicrobial resistance in East Africa. Afr J Lab Med. 2010;5(1):1-6.

23. Abebaw B, Dagnew M, Birhanemeskel T, Yeshitela B, Howe R, Abate E. Bacterial profile, antibiotic resistance pattern and associated factors from women attending postnatal health service at University of Gondar Teaching Hospital, Northwest Ethiopia. Infect Drug Resist. 2018;11:2169-78.

24. Bizimana K, Ndoli J, Bayingana C, Baluhe I, Gilson GJ, Habimana E. Prevalence and Risk Factors for Post Cesarean Delivery Surgical Site Infection in a Teaching Hospital Setting in Rural Rwanda: A Prospective Cross Sectional Study. Int J Curr Microbiol Appl Sci. 2016 Jun 15;5(6):631-41.

25. Mpogoro FJ, Mshana SE, Mirambo MM, Kidenya BR, Gumodoka B, Imirzalioglu C. Incidence and predictors of surgical site infections following caesarean sections at Bugando Medical Centre, Mwanza, Tanzania. Antimicrob Resist Infect Control. 2014 Aug 11;3(1).

26. Jido T, Garba I. Surgical-site infection following cesarean section in Kano, Nigeria. Ann Med Health Sci Res. 2012;2(1):33.

27. Bebell LM, Ngonzi J, Bazira J, Fajardo Y, Boatin AA, Siedner MJ, et al. Antimicrobial-resistant infections among postpartum women at a Ugandan referral hospital. PLoS One. 2017;12(4):1-13.

28. Workneh M, Katz M, Lamorde M, Cosgrove S, Manabe Y. Antimicrobial Resistance of Sterile Site Infections in Sub-Saharan Africa: A Systematic Review. Open Forum Infect Dis. 2017;4(4):1-5.

29. Sonderman KA, Nkurunziza T, Kateera F, Gruendl M, Koch R, Gaju E, et al. Using mobile health technology and community health workers to identify and refer caesarean-related surgical site infections in rural Rwanda: A randomised controlled trial protocol. BMJ Open. 2018;8(5):1-7.

30. Harris PA, Ph D, Taylor R, Thielke R, Ph D, Payne J, et al. Research Electronic Data Capture (REDCap) - A metadata-driven methodology and workflow process for providing translational research informatics support. J Biomed Inf. 2010;42(2):377-81.

31. Morisaki N, Ganchimeg T, Ota E, Vogel JP, Souza JP, Mori R, et al. Maternal and institutional characteristics associated with the administration of prophylactic antibiotics for caesarean section: a secondary analysis of the World Health Organization Multicountry Survey on Maternal and Newborn Health. BJOG. 2014;121 Suppl:66-75.

32. Sway A, Nthumba P, Solomkin J, Tarchini G, Gibbs R, Ren Y, et al. Burden of surgical site infection following cesarean section in sub-Saharan Africa: a narrative review. Int J Womens Health. 2019; Volume 11:309-18.

33. Elbur AI, Yousif MA, Sayed ASA El, Abdel-rahman ME. Misuse of prophylactic antibiotics and prevalence of postoperative wound infection in obstetrics and gynecology department in a Sudanese hospital. Health (Irvine Calif). 2014;6(2):158-64.

34. Okoth C, Opanga S, Okalebo F, Oluka M, Baker Kurdi A, Godman B. Point prevalence survey of antibiotic use and resistance at a referral hospital in Kenya: findings and implications. Hosp Pract (1995) 
[Internet]. 2018;46(3):128-36. Available from: https://doi.org/10.1080/21548331.2018.1464872

35. Huskins WC, Ba-Thike K, Festin MR, Limpongsanurak S, Lumbiganon P, Peedicayil A, et al. An international survey of practice variation in the use of antibiotic prophylaxis in cesarean section. Int $\mathrm{J}$ Gynecol Obstet. 2001;73(2):141-5.

Tables \& Figures

Table 1. Demographic and clinical characteristics of study participants $(\mathrm{N}=506)$

Table 2. Antibiotic prescriptions $(\mathrm{N}=506)$

Figure 1. Post-operative antibiotic prescriptions during hospitalisation

Figure 2. Discharge antibiotic prescriptions

\section{Hosted file}

Table_1.docx available at https://authorea.com/users/318049/articles/448040-gaps-betweenantibiotic-prescribing-practices-and-international-guidelines-for-women-undergoingcaesarean-deliveries-in-rural-rwanda-a-retrospective-cohort-study

\section{Hosted file}

Table_2.docx available at https://authorea.com/users/318049/articles/448040-gaps-betweenantibiotic-prescribing-practices-and-international-guidelines-for-women-undergoingcaesarean-deliveries-in-rural-rwanda-a-retrospective-cohort-study

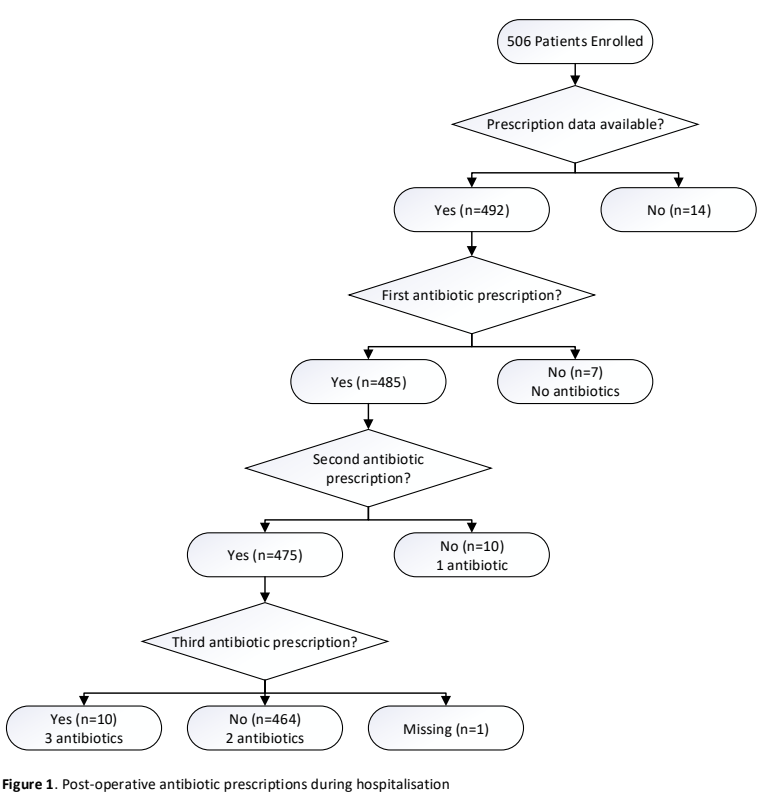




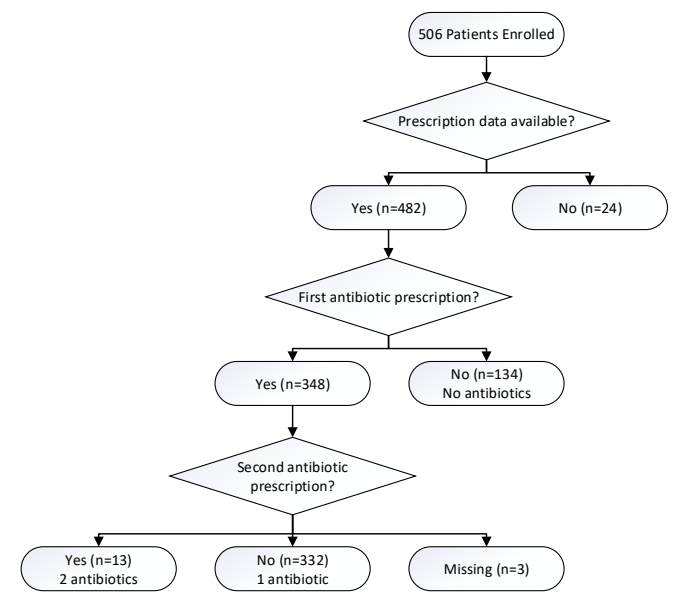

Figure 2. Discharge antibiotic prescriptions 